\title{
Purely Electronic and Vibronic Fluorescence Excitation of Single Terrylene Molecules in a Naphthalene Crystal
}

\author{
M. Banasiewicz, J. Dresner, O. Morawski, D. Wiącek
}

AND B. KOZANKIEWICZ*

Institute of Physics, Polish Academy of Sciences

al. Lotników 32/46, 02-668 Warsaw, Poland

Remembering Jurek Prochorow and many years in his group

\begin{abstract}
Fluorescence excitation spectra of spatially resolved single terrylene molecules in a naphthalene crystal were studied at $5 \mathrm{~K}$ as a function of exciting laser light intensity. The fully saturated fluorescence detection rate for purely electronic transition $(0,0)$ was found to be about twice smaller than that for the two main vibronic components located 239 and $251 \mathrm{~cm}^{-1}$ above. This relation is fulfilled when the triplet population rate $k_{23}$ is much weaker than the depopulation rate $k_{31}$. We propose that the weak fluorescence excitation lines observed at the frequencies of $38,67,97$, and $145 \mathrm{~cm}^{-1}$ correspond to nontotally symmetric low-frequency vibration modes of terrylene.
\end{abstract}

PACS numbers: 32.50.+d, 33.20.Kf, 33.50.Dq

\section{Introduction}

The study of single molecules, which has only recently become possible, eliminates ensemble averaging and offers access to statistical distributions, correlation and temporal fluctuations of macroscopic parameters. Thus it becomes a powerful technique for exploring the individual nanoscale behaviour of molecules in a complex local environment.

Single organic molecules are typically detected and studied in two distinct temperature ranges. One is the low temperature range, when the narrow zero-

*corresponding author; e-mail: kozank@ifpan.edu.pl 
-phonon lines of purely electronic transition $\left(S_{0} \rightarrow S_{1}\right)$ with high absorption cross-section allow to isolate single molecules spectrally by scanning the frequency of a single-mode laser. Second is the room temperature range, when molecules are usually excited to a vibronic level $\left(S_{0} \rightarrow S_{1}^{n}\right)$, fluorescence photons efficiently detected with the oil immersion microscope objectives and molecules isolated spatially with the aid of imaging techniques $[1,2]$. Not much work has been done at intermediate temperatures, between 10 and $290 \mathrm{~K}$ [3], where a manifold of thermally activated processes can operate. The reason is mainly due to experimental requirements. At elevated temperatures the zero-phonon lines are already replaced by the broad phonon wings and thus single molecules have to be isolated only spatially. Furthermore, an immersion oil freezes and in order to detect fluorescence photons one has to use the air gap version of a microscope objective. Thus, at intermediate temperatures the signal-to-noise ratio is poorer than that at room temperature.

In the present contribution we use our new, home-made confocal microscope, which is specially designed to study fluorescence of single molecules within the broad temperature range between 5 and $300 \mathrm{~K}$ [4-6]. An excitation source is a dye laser, which delivers picosecond pulses with the repetition rate of $76 \mathrm{MHz}$ and frequency band width $\approx 2 \mathrm{~cm}^{-1}$. Thus, we have to prepare our samples with a sufficiently low concentration of fluorescent molecules in order to be able to distinguish them spatially. Our laser can be scanned in the relatively broad frequency range providing opportunity to excite a single molecule to its purely electronic $S_{1}$ state as well as to some low-frequency vibrational modes of this state. It was the purpose of our work to verify experimentally how the excitation of different states influences the results. To achieve this goal we studied single terrylene $(\operatorname{Tr})$ molecules embedded into a sublimation grown naphthalene $(\mathrm{N})$ crystal. Single Tr molecules were already studied in deuterated $\left(\mathrm{C}_{10} \mathrm{D}_{8}\right) \mathrm{N}$ [7-9], as well as in protonated $\left(\mathrm{C}_{10} \mathrm{H}_{8}\right) \mathrm{N}$ crystals $[10,11]$ at cryogenic temperatures. All these previous studies were done however with the low-temperature single molecule spectroscopic technique by scanning a cw single-mode dye laser (with the band width $\approx 10^{-4} \mathrm{~cm}^{-1}$ ) and with many single Tr molecules within an excitation spot. Our new experimental approach provides opportunity to vary excitation intensity and for the first time we present purely electronic and vibronic transitions of a single Tr molecule saturated in the same frequency scan of the fluorescence excitation spectrum.

\section{Experimental}

Single crystals of zone-refined N slightly doped with $\operatorname{Tr}$ (purchased from the Institute für PAH-Forschung, Greifenberg, Germany) were grown by cosublimation of both components under a 800 mbar argon atmosphere. An extra heated small container with $\mathrm{Tr}$ allowed to increase and control the dopant concentration. Under these conditions we obtained good quality thin plates of $\mathrm{N}$ single crystals (thickness of a few tens of $\mu \mathrm{m}$ ) with the typical area of $\approx 10 \mathrm{~mm}^{2}$. 


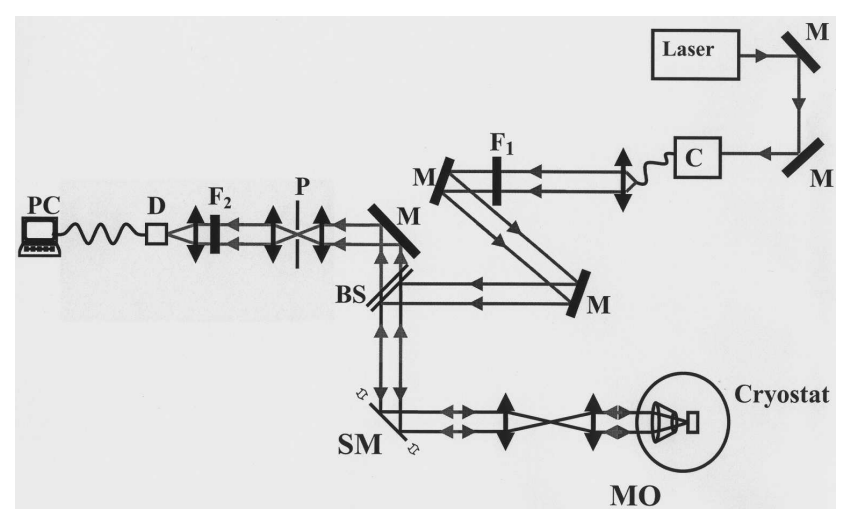

Fig. 1. Optical scheme of our confocal microscope operating within the temperature range between 5 and $300 \mathrm{~K} . \mathrm{M}$ - mirrors; C - fiber light collimator; F - filters; BS - beam splitter; SM - scanning mirror; MO - microscope objective; P — pinhole; $\mathrm{D}$ - photon counting module. Details in the text.

A crystal "flake" was inserted into a variable temperature optical cryostat which is part of a fluorescence scanning confocal microscope - see Fig. 1. The excitation source was a Coherent 700 dye laser (operating with Rh6G dye) pumped by a mode-locked Coherent Antares $76 \mathrm{Nd}$ :YAG laser. This laser created a train of 20 ps light pulses, each separated from the previous by 13 ns. The excitation beam was focused on the sample with the aid of a high aperture $(\mathrm{NA}=0.85)$ microscope objective MO (Bernhard Halle Nachfl. GmbH, Berlin) especially designed to operate from room temperature down to the liquid helium temperatures. Laser light spot had near-diffraction-limited diameter of about $500 \mathrm{~nm}$ (measured as the full width at half maximum). We were able to scan the laser light between 562 and $578 \mathrm{~nm}$ exciting $\operatorname{Tr}$ molecules to their $S_{1}$ state via purely electronic $(0,0)$ transition or to the vibronic components located some $250 \mathrm{~cm}^{-1}$ above. We estimated that the band width of our laser is $\approx 2 \mathrm{~cm}^{-1}$ and precision of the frequency reading $\pm 2 \mathrm{~cm}^{-1}$. Sample holder with the microscope objective was located inside an optical cryostat whose temperature could be stabilised between 5 and $300 \mathrm{~K}$. The spot position on the sample was capable of coarse mechanical adjustment in a horizontal plane and along the optical axis, and of precise adjustment by tilting a laser beam in respect of the objective by means of the system composed of a galvo-scanner SM (Cambridge Tech., model 6230) and telecentrical arrangement of two lenses located outside the cryostat. The scanned area in the latter case was $30 \times 30 \mu \mathrm{m}^{2}$. The fluorescence photons were separated from the scattered excitation light with the aid of a dichroic beam-splitter BS (Chroma Tech., QP585lp). Emission beam was further filtered in frequency by using a band-pass (Chroma Tech., HQ645/110) together with a long-pass (RG610) filter F2 and spatially by focusing it on a pinhole $\mathrm{P}$ (30 $\mu \mathrm{m}$ diameter). Fluorescence photons were detected with a photon counting module D (EG\&G SPCM-AQ141) and acquired with the 
aid of a multi-channel scaler card (LightScan) inserted into a PC. Typical signal-to-noise ratio, when light was focused on a single Tr molecule, varied between 5 and 20 , depending on a molecule and temperature.

Single molecule fluorescence decay times were measured with the "time-correlated" single photon counting technique. During this experiment the repetition rate of a laser system was reduced to $7.6 \mathrm{MHz}$. We used "inverted configuration", where the signal "start" was provided by a fluorescence photon detected with a photon counting module D, whereas the signal "stop" by the exciting molecule laser light pulse monitored with an avalanche photodiode. Other components of the setup were a Tennelec TC 454 quad constant fraction discriminator, a TC 864 time-to-amplitude converter, and a Nucleus PCA-II multichannel analyser.

\section{Results and discussion}

Figure 2 presents the $15 \times 15 \mu \mathrm{m}^{2}$ fluorescence image obtained at $7 \mathrm{~K}$, where the bright spots correspond to single Tr molecules embedded in an $\mathrm{N}$ crystal. To study any selected single molecule in the image we focused the microscope objective on its spot.

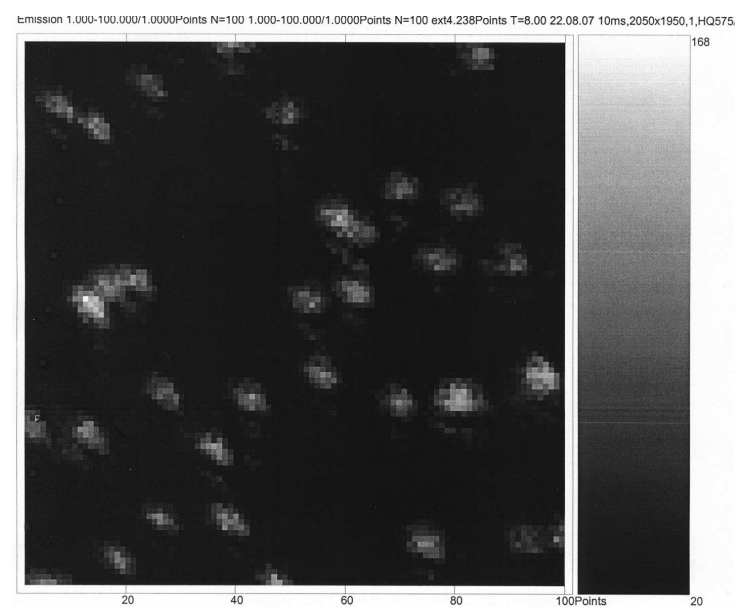

Fig. 2. Confocal fluorescence image $\left(15 \times 15 \mu \mathrm{m}^{2}\right)$ of single $\mathrm{Tr}$ molecules embedded in an $\mathrm{N}$ crystal at $5 \mathrm{~K}$. $\lambda_{\mathrm{exc}}=566.6 \mathrm{~nm}$. Bright spots correspond to single Tr molecules. The grey scale on the right side of the figure gives number of photon counts in $10 \mathrm{~ms}$.

The fluorescence excitation spectrum of a selected single Tr molecule at $5 \mathrm{~K}$ was measured when scanning the wavelength of a dye laser. The set of spectra, measured between 565 and $576 \mathrm{~nm}$ for typical molecule at various excitation intensities, is shown in Fig. 3. The line at $574.4 \mathrm{~nm}\left(17405 \pm 3 \mathrm{~cm}^{-1}\right)$ corresponds to the $(0,0)$ transition whereas the two other lines, at $566.6 \mathrm{~nm}\left(17645 \pm 2 \mathrm{~cm}^{-1}\right)$ and $566.2 \mathrm{~nm}\left(17658 \pm 2 \mathrm{~cm}^{-1}\right)$, are the vibronic components separated by $239 \pm 2$ and $251 \pm 2 \mathrm{~cm}^{-1}$ from the $(0,0)$ origin. The full width at half maximum of all 


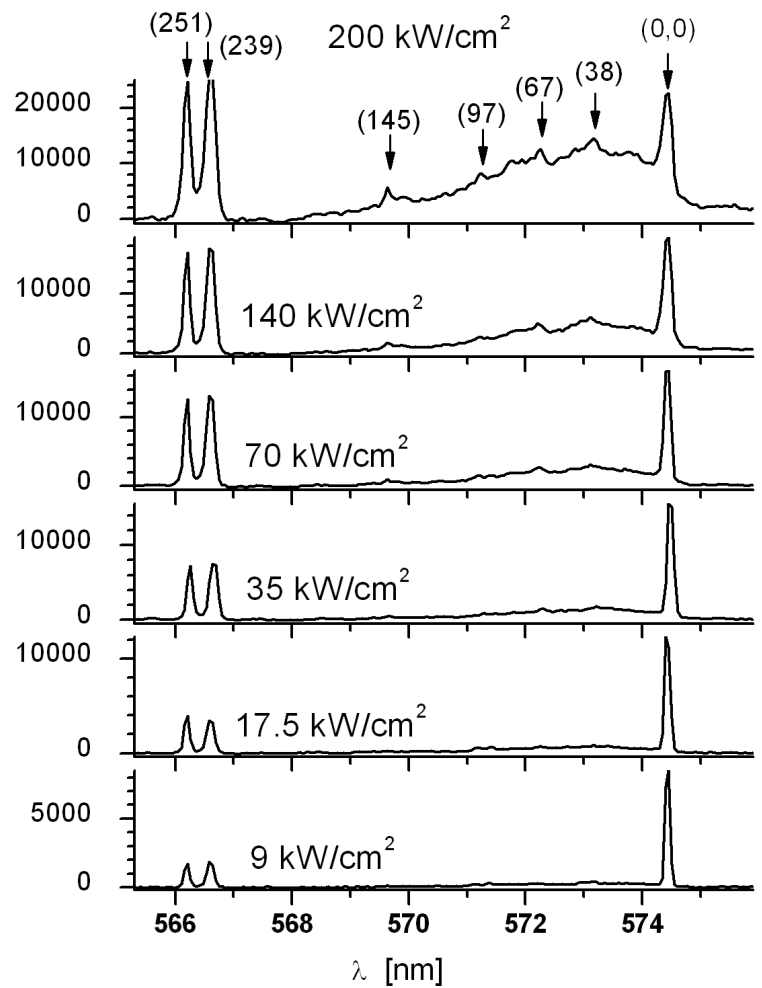

Fig. 3. Fluorescence excitation spectra of typical single $\operatorname{Tr}$ molecule in an $\mathrm{N}$ crystal at $5 \mathrm{~K}$ recorded at different excitation intensities. The fluorescence intensity given in counts-per-second (cps). The numbers in parentheses indicate the vibronic frequencies (in $\mathrm{cm}^{-1}$ ) obtained with respect to the origin $(0,0)$ line.

the lines detected at the lowest exciting light intensity is about $2 \mathrm{~cm}^{-1}$, and since the zero-phonon line width is much more narrow $\left(\approx 2 \times 10^{-3} \mathrm{~cm}^{-1}\right.$ at $\left.1.5 \mathrm{~K}[11]\right)$ this value indicates the spectral line width of our laser. All of the above lines are of the zero-phonon nature. Weakly structured phonon side band accompany the $(0,0)$ origin line at the limit of high excitation intensity, only. Such the behaviour clearly indicates that the dynamic interactions between the guest, $\mathrm{Tr}$, and the host, $\mathrm{N}$, crystal cage are weak.

The above spectra are in good agreement with those observed for single Tr molecules in a naphthalene- $\mathrm{d}_{8}$ crystal at 1.4-1.8 K [7-9]. In these previous studies, performed with a single-mode dye laser excitation, one spectroscopic site with maximum at $574.3 \mathrm{~nm}$ and inhomogeneous band width of about $7 \mathrm{~cm}^{-1}$ [7], and two vibronic frequencies, $\nu_{1}$ at 237 and $\nu_{2}$ at $249 \mathrm{~cm}^{-1}$ above the purely electronic band were observed [9]. Concentration of Tr molecules in our N crystals was much lower than in the previous work, and we studied purely electronic and vibronic components of the fluorescence excitation spectrum of each individual 
single molecule separately, without the need to use more complicated pump-probe approach [9]. Following Kummer et al. interpretation [12], the first vibronic band, $\nu_{1}$, should be assigned to a totally symmetric long-axes stretch vibration, whereas the second, $\nu_{2}$, to a torsional vibration which indicates non- planar distortion of Tr molecule by the crystalline matrix.

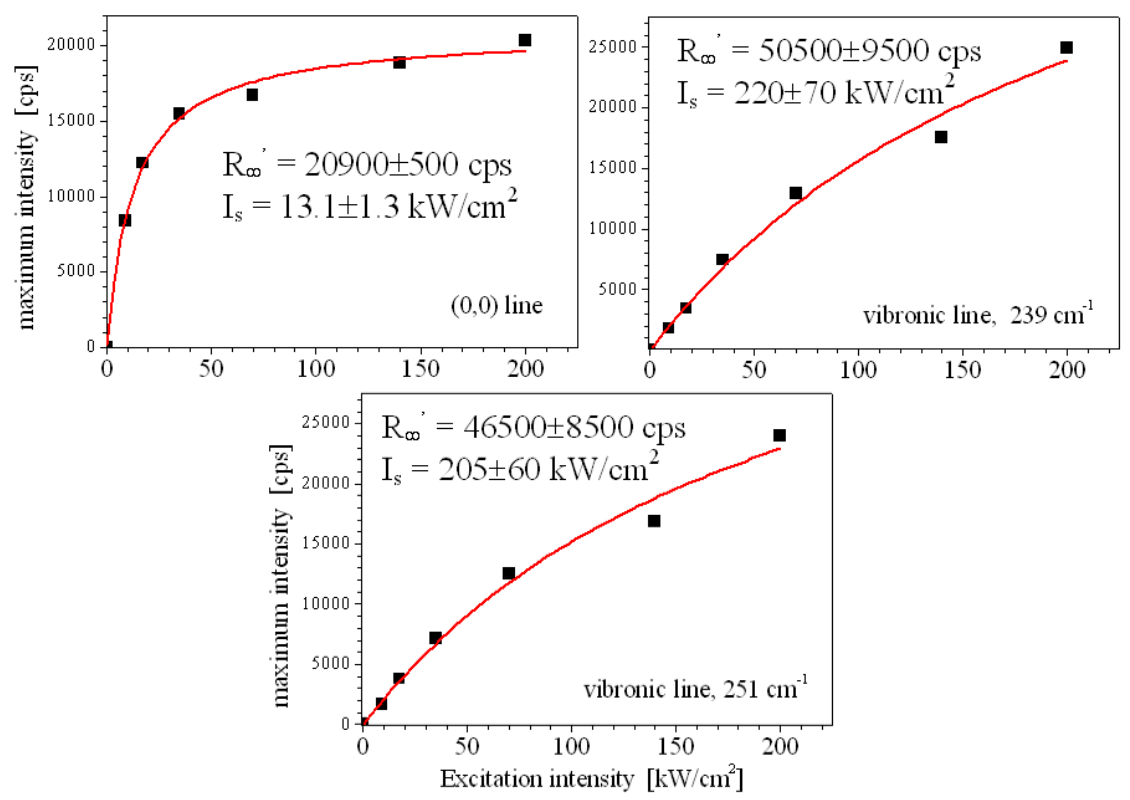

Fig. 4. Maximum intensity of the fluorescence excitation lines of a single Tr molecule in an $\mathrm{N}$ crystal (Fig. 3) as a function of excitation light intensity for the $(0,0)$ line, and for the vibronic lines separated by 239 and $251 \mathrm{~cm}^{-1}$ from the $(0,0)$ origin. Fits were done according to formula (1), with the fitting parameters indicated.

The characteristic feature of the fluorescence excitation spectrum is strong, nonlinear dependence of the line intensities on the exciting light intensity - see Fig. 4. To discuss this dependence we used the saturation formula [1]:

$$
R(I)=R_{\infty}\left(I / I_{\mathrm{s}}\right) /\left[1+\left(I / I_{\mathrm{s}}\right)\right]
$$

where $I_{\mathrm{S}}$ is the saturation intensity, $R(I)$ - the fluorescence detection rate at intensity $I$, and $R_{\infty}$ the fully saturated fluorescence detection rate. The fitting parameters, $R_{\infty}^{\prime}$ and $I_{\mathrm{s}}$, obtained for the $(0,0)$ origin and two main vibronic lines of the molecule whose spectra are presented in Fig. 3 are given on the right side of the corresponding figure. It should be pointed out that experimentally we measure parameter $R_{\infty}^{\prime}=\eta R_{\infty}$, where $\eta$ is the detection efficiency of a setup, which is presently independent of a wavelength of excitation.

It can be easily seen that the fully saturated detection rate $R_{\infty}$ for the molecule excited to its purely electronic (0 vibration) state is approximately half 
of that when molecule is excited to any of the vibronic levels. To explain, we have to take into account that in the former case populations of the $S_{1}$ and $S_{0}$ states under full saturation can be only equal, whereas in the former case the $S_{0}$ state can be completely emptied in favour of the population of the $S_{1}$ state. Thus, the formulas for the fully saturated detection rate are different, and it is [1]:

$$
R_{\infty}^{00}=k_{21}^{\mathrm{r}} /\left(2+k_{23} / k_{31}\right),
$$

for excitation to the 0 vibronic level, and

$$
R_{\infty}^{0 v}=k_{21}^{\mathrm{r}} /\left(1+k_{23} / k_{31}\right),
$$

for excitation to any of the vibronic components. Here, $k_{21}^{\mathrm{r}}$ is the radiative decay rate of the $S_{1}$ state, and $k_{23}$ and $k_{31}$ are the population and depopulation rates of the triplet $T_{1}$ state, respectively. According to the formulas (2) and (3), $R_{\infty}^{0 v} \approx 2 R_{\infty}^{00}$ when $k_{23} \ll k_{31}$. The triplet population and depopulation rates can be determined from the fluorescence intensity correlation function [13]. Such studies were already performed for single Tr molecules in a $p$-terphenyl crystal, and the relation $k_{23} \ll k_{31}$ was found $[4,14]$.

The saturation intensity $I_{\mathrm{s}}$ is inversely proportional to the absorption cross-section [1] of considered transition, but it is also dependent on the relative widths of the absorption and excitation lines. Since the spectral line width of our excitation laser is much broader than the real widths of the absorption lines it is difficult to discuss in more detail the obtained values of the saturation intensity.

We should add that the above discussion of the saturation effect bases on the three level scheme, which takes into account the transitions between the ground, $S_{0}$, and two excited, $S_{1}$ and $T_{1}$, states, only. One can argue that when we work with the excitation intensity above the saturation level (which is realised for the purely electronic transition) we should consider a more complicated model with contributions from the $S_{1} \rightarrow S_{n}$ and $T_{1} \rightarrow T_{n}$ absorption pathways as well. Simple kinetic consideration led us however to the conclusion that the above pathways can be neglected in our case. Activation of the absorption $S_{1} \rightarrow S_{n}$ pathway followed by the intersystem crossing to triplet manifold should lead to a continuous decrease in the fluorescence intensity at the high excitation intensity limit - which was not observed in our experiment. Contribution from the $T_{1} \rightarrow T_{n}$ absorption can be neglected because population of the $T_{1}$ state is very small (due to $k_{23} \ll k_{31}$ ).

Unexpected and interesting was observation of weak lines separated from the $(0,0)$ electronic origin by $39,67,97$, and $145 \mathrm{~cm}^{-1}$. These lines were superimposed on a broad phonon-side band accompanying purely electronic transition monitored at the highest excitation intensities. We studied several single Tr molecules and for all of them we observed these weak lines (with the experimental frequency reading precision of $\pm 2 \mathrm{~cm}^{-1}$ ). Recently, the set of low-frequency modes (with the maxima at $56.6,106.2,160.6$, and $199.4 \mathrm{~cm}^{-1}$ ) observed in the bulk fluorescence excitation spectrum of $\operatorname{Tr}$ in a supersonic jet expansion was attributed to nontotally symmetric vibronic transitions [15]. We postulate the same origin of the mentioned weak 
lines observed in our experiment. Nontotally symmetric vibronic transitions are usually absent (or have extremely low intensity if to compare with the intensity of the totally symmetric transitions) in a typical spectrum of the aromatic hydrocarbon and one has to perform experiment in special conditions, like saturation of the dominating lines in the spectrum, in order to be able to observe them. In addition to the above lines we observed also some other weak lines separated from the $(0,0)$ origin by less than $100 \mathrm{~cm}^{-1}$, but the frequencies of these lines were not reproduced for different single molecules. Therefore, we postulate that these extra lines are fingerprints of the local phonon modes characterising environment of each individual guest molecule.

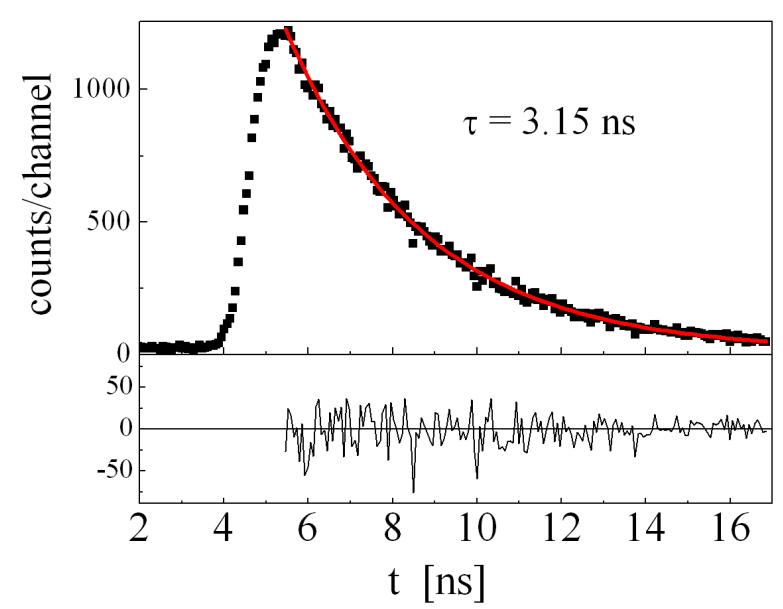

Fig. 5. Typical fluorescence decay curve of single Tr molecule in an $\mathrm{N}$ crystal at $5 \mathrm{~K}$. $\lambda_{\text {exc }}=566.6 \mathrm{~nm}$. The residual plot for least-squares fit to single exponential dependence is given below the decay curve.

Fluorescence decay curves were measured for several single Tr molecules in the $\mathrm{N}$ crystal, and typical curve is shown in Fig. 5. Fits of all of the curves to the single exponential dependence were perfect and we got the decay times varying between 3.1 and $3.2 \mathrm{~ns}$ for different molecules. This value is the same, within the experimental error of $\pm 0.1 \mathrm{~ns}$, as that measured previously with our "timecorrelated" single photon counting setup for the bulk sample of Tr in an anthracene crystal [11]. We have to mention however, that we expected slightly longer decay time, close to $4 \mathrm{~ns}$, when taking into account the data collected in Ref. [16] for the bulk samples of $\operatorname{Tr}$ in different solids.

The fluorescence quantum yield of Tr is very high, close to unity [17], and therefore the reciprocal of the fluorescence decay time gives the radiative lifetime of the $S_{1}$ state of $\operatorname{Tr}$ molecules, $k_{21}^{\mathrm{r}}=(3.2 \pm 0.1) \times 10^{8} \mathrm{~s}^{-1}$. Now, using the formula (2) or (3), with $k_{23} \ll k_{31}$, we can estimate the fully saturated detection rate $R_{\infty}^{00}=(1.6 \pm 0.1) \times 10^{8} \mathrm{~s}^{-1}$, and $R_{\infty}^{0 v}=(3.2 \pm 0.1) \times 10^{8} \mathrm{~s}^{-1}$. One should, however, 
realise that the formulas (2) and (3) were derived for a cw laser excitation, but in our experiment we used a model-locked laser which provided light pulses with the repetition $0.76 \times 10^{8} \mathrm{~s}^{-1}(76 \mathrm{MHz})$. Thus, the fully saturated detection rates in our case were about $4.2(=3.2 / 0.76)$ times smaller than they would be with a cw excitation. Taking into account the experimentally determined $R_{\infty}^{\prime}$ values we estimated the detection efficiency $\eta \approx 10^{-3}$. This is a typical value for the detection efficiency in a low temperature experiment. We were able to improve the number of detected fluorescence photons when playing with an experimental setup (using different filters and diameters of pinhole) but we observed the parallel, stronger increase in the background signal (and noise).

\section{Summary}

We studied fluorescence excitation spectra of single Tr molecules in an $\mathrm{N}$ crystal at $5 \mathrm{~K}$ in the spectral range which covers the purely electronic transition at $17405 \pm 3 \mathrm{~cm}^{-1}$ and two main vibronic components located 239 and $251 \mathrm{~cm}^{-1}$ above. For the first time in single molecule studies we measured these spectra at different excitation intensities and found that the fully saturated fluorescence detection rate for purely electronic transition is about twice smaller than that for any of the vibronic components. Such relation reflects different population rates of the $S_{1}$ state and can be strictly fulfilled when the triplet $T_{1}$ state population rate $k_{23}$ is much smaller than the depopulation rate $k_{31}$.

In the spectra detected at high excitation intensity we observed some weak fluorescence excitation lines separated by $38,67,97$, and $145 \mathrm{~cm}^{-1}$ from the $(0,0)$ origin line. These lines were present in the spectra (measured in appropriate conditions) of the majority of studied single molecules and thus, we propose that they are nontotally symmetric low frequency vibration modes of $\mathrm{Tr}$, recently observed in a supersonic jet expansion [15].

We measured also fluorescence decay time of single $\mathrm{Tr}$ molecules in an $\mathrm{N}$ crystal $(\approx 3.15 \mathrm{~ns}$ ), and determined the theoretical value of the fully saturated detection rate with a cw laser excitation. Since we used a mode-locked laser with the $76 \mathrm{MHz}$ repetition this rate was about 4 times smaller. Estimated detection efficiency of fluorescence photons in our experiment was $\eta \approx 10^{-3}$.

\section{Acknowledgments}

This work was partly supported by the Ministry of Science and Higher Education, research project N202 051 32/1188.

\section{References}

[1] Th. Basche, W.E. Moerner, M. Orrit, U.P. Wild, Single-Molecule Optics Detection, Imaging and Spectroscopy, VCH, Weinheim 1996.

[2] R. Rigler, M. Orrit, T. Basche, Single Molecule Spectroscopy. Nobel Conference Lectures, Springer, Berlin 2001. 
[3] W. Göhde, J. Tittel, Th. Basche, C. Bräuchle, U.C. Fischer, H. Fuchs, Rev. Sci. Instrum. 68, 2466 (1997).

[4] M. Banasiewicz, O. Morawski, D. Wiạcek, B. Kozankiewicz, Chem. Phys. Lett. 414, 374 (2005).

[5] M. Banasiewicz, D. Wiącek, B. Kozankiewicz, Chem. Phys. Lett. 425, 94 (2006).

[6] M. Banasiewicz, D. Wiạcek, B. Kozankiewicz, Chem. Phys. Lett. 425, 289 (2006).

[7] H. Bach, A. Renn, U.P. Wild, Single Mol. 1, 73 (2000).

[8] T. Nonn, T. Plakhotnik, Chem. Phys. Lett. 336, 97 (2001).

[9] T. Plakhotnik, T. Nonn, V. Palm, Chem. Phys. Lett. 357, 397 (2002).

[10] M.A. Kol'chenko, B. Kozankiewicz, A. Nicolet, M. Orrit, Opt. Spectrosc. 98, 681 (2005).

[11] A. Nicolet, M.A. Kol'chenko, B. Kozankiewicz, M. Orrit, J. Chem. Phys. 124, 164711 (2006).

[12] S. Kummer, F. Kulzer, R. Kettnet, T. Basche, C. Tietz, C. Glowatz, C. Kryschi, J. Chem. Phys. 107, 7673 (1997).

[13] J. Bernard, L. Fleury, H. Talon, M. Orrit, J. Chem. Phys. 98, 850 (1993).

[14] A.C.J. Brouver, E.J.J. Groenen, J. Schmidt, Phys. Rev. Lett. 80, 3944 (1998).

[15] I. Deperasinska, A. Zehnacker, F. Lahmani, P. Borowicz, J. Sepioł, J. Phys. Chem. A 111, 4252 (2007).

[16] G.S. Harms, Th. Irngartinger, D. Reiss, A. Renn, U.P. Wild, Chem. Phys. Lett. 313, 533 (1999).

[17] M. Banasiewicz, I. Deperasińska, D. Fabjanowicz, B. Kozankiewicz, Chem. Phys. Lett. 356, 541 (2002). 